\title{
EDITORIAL
}

\section{It is Time to Change How We Measure Blood Pressures in the Office}

Lee A. Green, MD, MPH

Family Medicine, University of Alberta, Edmonton, Alberta, Canada
$\mathrm{F}$ or a typical patient in my practice with Stage I hypertension and 1 or more other cardiovascular risk factors, the number needed to treat (NNT) for 5 years to prevent a death, acute coronary event, stroke, heart failure, or renal failure is only 11 , and patients at higher risk (especially those with known coronary or renal disease) benefit even more. ${ }^{1,2}$ With such a large benefit for outcomes that matter so much to patients, and a condition so many patients have, treating hypertension properly is clearly one of the most important things we do in family medicine.

We have to measure blood pressure accurately to treat it properly. In practice that's always been a problem. Our evidence base for hypertension treatment is clinical trials that used standardized office blood pressure (SOBP) measurement. SOBP requires the patient be seated at rest for 5 minutes, with feet on the floor, back supported, and arm supported at mid-chest height. When giving continuing medical education talks, I find that describing SOBP and asking the audience, "Is it always done that way in your practice?" reliably produces laughter.

Worse, even when done properly, SOBP is neither especially consistent and repeatable ${ }^{3}$ nor the best predictor of outcomes. ${ }^{4}$ White-coat hypertension is common, and can mislead us into overtreating blood pressure.

Is that bad? Some believe that white-coat hypertension, although less dangerous than sustained hypertension, still leads to more adverse outcomes than normotension and thus does merit treatment. ${ }^{5,6}$

Conflicts of interest: author reports none.

\section{CORRESPONDING AUTHOR}

Lee A. Green, MD, MPH

8440112 St, NW

Family Medicine

University of Alberta

Edmonton, Alberta, Canada T6G 2R7

lagreen@ualberta.ca
Franklin et $\mathrm{al}^{7}$, however, recently published a more careful analysis demonstrating that when age and risk are properly matched, white-coat hypertension patients do not have worse outcomes than similar normotensive patients. Overdiagnosing hypertension can have costly financial and social consequences for patients, and overtreating imposes annoying burden (and cost) even on those who have hypertension. Nor can we assume overtreatment is medically harmless. Overtreating is essentially unknowingly treating to a more aggressive than standard level of blood pressure reduction, and the SPRINT trial ${ }^{8}$ demonstrated a substantial rate of serious adverse events from doing so. Those adverse events may be worth risking for the high-risk patients who meet the inclusion criteria for SPRINT, but imposing the harms of overtreatment on low-risk patients-or those who do not even have hypertension at all-is unjustifiable.

So how do we avoid overtreatment? The gold standard is 24-hour ambulatory blood pressure monitoring. While it certainly has a place, however, it is somewhat costly and cumbersome even for initial diagnosis. For routine monitoring it would be quite impractical. Home monitoring can be very useful for those patients motivated to do it reliably, but what about everyone else?

Fortunately, over the last 15 years or so a significant body of evidence has emerged in primary care practices on automated office blood pressures collected with patients sitting undisturbed 6 times over 5 to 10 minutes (AOBP) $)^{4,9,10}$ or every 5 minutes for 30 minutes (OBP30). ${ }^{3,11} \mathrm{AOBP}$ and $\mathrm{OBP} 30$ are superior to routine office blood pressure measurement and to SOBP, closely approximating (for daytime pressures, at least) continuous ambulatory monitoring. Both methods could allow us to avoid overdiagnosis and overtreatment. Do they, in practice?

In this issue, Bos \& Buis $^{12}$ take the next necessary step. They provide evidence that treatment decisions change for a significant number of patients when OBP30 is used, compared to routine office blood pressures. At 
this point the evidence seems clear: routine office blood pressures, and even SOBP, should no longer be used to diagnose hypertension nor to adjust treatment.

So what are the implications for practice change? Aside from the obvious equipment needs, we will need to change our thinking on diagnosis and treatment thresholds, choose a method, and implement our choice properly. A well-done practice-based research network study comparing AOBP and OBP30 is an important first step. Since SPRINT used an abbreviated AOBP, with just 3 measurements, adding that to the comparison would be valuable. We need to know whether AOBP or abbreviated AOBP, done in a few minutes, are just as good as a 30-minute protocol, or if there is a convenience/accuracy tradeoff we need to consider.

We also will need to rethink our thresholds. The threshold for Stage 1 hypertension of 140/90 is drawn from studies using SOBP. Home and continuous ambulatory blood pressures run lower, and as AOBP and OBP30 closely approximate daytime ambulatory pressures, using 140/90 would result in underdiagnosis and undertreatment. Myers et $\mathrm{al}^{13}$ have provided observational evidence based on patient-oriented outcomes for $135 / 85$, at least for older adults. Replication and extension to younger populations, and to patients with higher risk profiles, is needed. Ideally the evidence for practice should come from practice-again suggesting that practice-based research networks are the best laboratories.

Changing how we do things in our offices may be a challenge too. Both AOBP (whether standard or abbreviated) and OBP30 require setting a patient up and then leaving them undisturbed while the measurements are taken. If we are to avoid overdiagnosing and overtreating due to the white-coat effect, we "white coats" need to stay out of the room! (Taking off the coat won't fool anyone's sympathetic nervous system, either.) For some practices that will be easy, but for some of us it will mean at least changing our routines, and sometimes even rearranging our offices. Getting it right will be a good use for our teams' quality improvement skills. Because hypertension treatment matters so much to so many, we owe it to our patients to get it right.
To read or post commentaries in response to this article, see it online at http://www.annfammed.org/content/15/2/105.

Key words: hypertension; blood pressure determination; white coat hypertension; practice patterns, physicians'

\section{References}

1. Chobanian AV, Bakris GL, Black HR, et al; National Heart, Lung, and Blood Institute Joint National Committee on Prevention, Detection, Evaluation, and Treatment of High Blood Pressure; National High Blood Pressure Education Program Coordinating Committee. The Seventh Report of the Joint National Committee on Prevention, Detection, Evaluation, and Treatment of High Blood Pressure: the JNC 7 report. JAMA. 2003;289(19):2560-2572.

2. Ogden LG, He J, Lydick E, Whelton PK. Long-term absolute benefit of lowering blood pressure in hypertensive patients according to the JNC VI risk stratification. Hypertension. 2000;35(2):539-543.

3. Scherpbier-de Haan N, van der Wel M, Schoenmakers G, et al. Thirty-minute compared to standardised office blood pressure measurement in general practice. Br J Gen Pract. 2011;61(590):e590-e597.

4. Beckett L, Godwin M. The BpTRU automatic blood pressure monitor compared to 24 hour ambulatory blood pressure monitoring in the assessment of blood pressure in patients with hypertension. BMC Cardiovasc Disord. 2005;5(1):18.

5. Briasoulis A, Androulakis E, Palla M, Papageorgiou N, Tousoulis D. White-coat hypertension and cardiovascular events: a meta-analysis. J Hypertens. 2016;34(4):593-599.

6. Mancia G. Clinical significance of white-coat hypertension. J Hypertens. 2016;34(4):623-626.

7. Franklin SS, Thijs L, Asayama K, et al; IDACO Investigators. The cardiovascular risk of white-coat hypertension. J Am Coll Cardiol. 2016;68(19):2033-2043.

8. Wright JT Jr, Williamson JD, Whelton PK, et al; SPRINT Research Group. A randomized trial of intensive versus standard bloodpressure control. N Engl J Med. 2015;373(22):2103-2116.

9. Myers MG, Kaczorowski J, Dawes M, Godwin M. Automated office blood pressure measurement in primary care. Can Fam Physician. 2014;60(2):127-132.

10. Myers MG. A short history of automated office blood pressure - 15 years to SPRINT. J Clin Hypertens (Greenwich). 2016;18(8):721-724.

11. van der Wel MC, Buunk IE, van Weel C, Thien TABM, Bakx JC. A novel approach to office blood pressure measurement: 30-minute office blood pressure vs daytime ambulatory blood pressure. Ann Fam Med. 2011;9(2):128-135.

12. Bos MJ, Buis S. Thirty-minute office blood pressure monitoring in primary health care. Ann Fam Med. 2017;15(2):120-123.

13. Myers MG, Kaczorowski J, Paterson JM, Dolovich L, Tu K. Thresholds for diagnosing hypertension based on automated office blood pressure measurements and cardiovascular risk. Hypertension. 2015;66(3):489-495. 